\title{
Epitaxial insulator for bottom-gate field-effect devices based on $\mathrm{TiO}_{2}$
}

\author{
Masao Katayama $^{1,2}$, Hideomi Koinuma ${ }^{2,3,4}$,Yuji Matsumoto ${ }^{1,2, *}$ \\ ${ }^{1}$ Materials and Structures Laboratory, Tokyo Institute of Technology \\ 4259 Nagatsuta, Midori-ku, Yokohama 226-8503, Japan \\ ${ }^{2}$ CREST, Japan Science and Technology Corporation \\ 4-1-8 Honcho, Kawaguchi-shi, Saitama 332-0012, Japan \\ ${ }^{3}$ Graduate School of Frontier Sciences, The University of Tokyo \\ 5-1-5, Kashiwanoha, Kashiwa, Chiba 277-8568, Japan \\ ${ }^{4}$ National Institute for Material Science, \\ 1-2-1 Sengen, Tsukuba, Ibaraki 305-0047, Japan \\ e-mail : matsumoto1@oxide.msl.titech.ac.jp
}

\section{Abstract}

Field-effect devices with bottom-gate structure based on anatase $\mathrm{TiO}_{2}$ active channels were fabricated. The key factor to achieve this was the quality of epitaxial insulator layers, viz.; the insulating property and their crystallinity, because they work not only as a well insulator for field-effect devices but also as a template for the subsequent growth of epitaxial active layers. Our devices showed typical transistor actions. On-to-off current ratio exceeded $10^{4}$ and the field effect mobility of $0.04 \mathrm{~cm}^{2} / \mathrm{Vs}$ were obtained. Interestingly, the device characteristics were found to be sensitive to ambient and light, suggesting their potential for manipulating the fruitful $\mathrm{TiO}_{2}$ surface functions by tuning the gate voltage.

Keyword: Electric field effect, epitaxy of thin film, Titanium dioxide

(C) 2007. This manuscript version is made available under the Elsevier user license http://www.elsevier.com/open-access/userlicense/1.0/ 


\section{Introduction}

Recent development of oxide electronics attracts much attention from both basic and applied viewpoints due to the wide variety of their physical and chemical properties [1]. Field-effect technique with oxide active channel has been applied for the control of oxide physical properties through their carrier concentration [2-8]. This enables to control carrier concentrations cleanly and reversively. We, indeed, have already reported that field-effect transistors based on $\mathrm{TiO}_{2}$ active channels with top-gate device structure and showed the potential for the carrier control of $\mathrm{TiO}_{2}$ by field-effect [9]. In order to utilize fruitful surface chemical phenomena of $\mathrm{TiO}_{2}[10]$, e.g., photocatalytic properties and photo-induced hydrophilic conversion, field-effect devices with exposed $\underline{\mathrm{TiO}}_{2} \underline{\text { active surface }}$ are required, i.e., bottom-gate device structure. To achieve this, one of the key requirements is the epitaxial insulator. It is required to fulfill both insulating properties for field-effect device and high crystallinity for the template of the subsequent growth of epitaxial active layers. In this study we have reported the fabrication of field-effect devices with bottom-gate structure based on anatase $\mathrm{TiO}_{2}$ channels and shown their potential for the tuning of surface functions by the gate voltage.

\section{Experimental}

Our device structure $\underline{\text { is }}$ schematically illustrated in Fig.1(a). Nb-doped $\underline{(0.5}$ $\underline{\mathrm{wt}} \%$ ) $\mathrm{SrTiO}_{3}$ single crystals (001) (available from Shinkosha Co.) were used as substrates, which worked as a gate electrode. Epitaxial $\mathrm{LaAlO}_{3}$ layers, which worked as an insulator, 
were grown by pulsed laser deposition (PLD). A $\mathrm{LaAlO}_{3}$ single crystal was ablated by $\mathrm{KrF}$ excimer laser $\left(\lambda=248 \mathrm{~nm}, \sim 1.5 \mathrm{~J} / \mathrm{cm}^{2}, 5 \mathrm{~Hz}\right)$ to deposit films onto a substrate kept at $700^{\circ} \mathrm{C}$. Oxygen partial pressure during $\mathrm{LaAlO}_{3}$ growth was varied from $1 \times 10^{-5}$ Torr to 1 x $10^{-2}$ Torr. Subsequent to the $\mathrm{LaAlO}_{3}$ growth, anatase $\mathrm{TiO}_{2}$ was epitaxially grown also by PLD. Substrate temperature, repetition rate and laser fluence of $\mathrm{KrF}$ excimer laser, and oxygen partial pressure were $650^{\circ} \mathrm{C}, 5 \mathrm{~Hz}$ and $\sim 1.5 \mathrm{~J} / \mathrm{cm}^{2}, 1 \times 10^{-3}$ Torr, respectively. After $\mathrm{TiO}_{2}$ growth, the sample was post annealed at $400^{\circ} \mathrm{C}$ for $2 \mathrm{~h}$ in atomospheric $\mathrm{O}_{2}$. Finally, $\underline{\mathrm{Al}}$ electrodes were formed by vacuum evaporation. They were patterned through stainless steel stencil masks $(\mathrm{W} / \mathrm{L}=500 \mu \mathrm{m} / 200 \mu \mathrm{m})$. Typical thicknesses of $\mathrm{LaAlO}_{3}$, $\mathrm{TiO}_{2}$, and $\mathrm{Al}$ were $500 \mathrm{~nm}, 10 \mathrm{~nm}, 20 \mathrm{~nm}$, respectively. Transistor characteristics were evaluated with HP4155B semiconductor parameter analyzer at room temperature in various ambient.

\section{Results and discussions}

Figures 1(b)-(d) show reflection high-energy electron diffraction (RHEED) pattern, atomic force microscope (AFM) image and X-ray diffraction (XRD) patterns of $\mathrm{LaAlO}_{3}$ epitaxial insulator grown at oxygen partial pressure of $1 \times 10^{-4}$ Torr. The streak pattern of RHEED shows that $\mathrm{LaAlO}_{3}$ film grew epitaxially in plane, although the pattern was relatively diffuse. The XRD pattern shows c-axis oriented $\mathrm{LaAlO}_{3}$. The full width of half maximum of the rocking curve of $\mathrm{LaAlO}_{3}(002)$ was $0.7^{\circ}$. RHEED and XRD results clearly shows that $\mathrm{LaAlO}_{3}$ films grow epitaxially on $\mathrm{Nb}: \mathrm{SrTiO}_{3}(001)$, with a fairly good crystallinity of $\mathrm{LaAlO}_{3}$ film. The AFM image of $\mathrm{LaAlO}_{3}$ surface reveal a smooth 
morphology with root mean square roughness of about $1 \mathrm{~nm}$, although the surface morphology was not atomically flat. These results further support an epitaxial growth of $\mathrm{LaAlO}_{3}$. Similar quality of the $\mathrm{LaAlO}_{3}$ films grown at oxygen partial pressure of $1 \times 10^{-3}$ Torr and $1 \times 10^{-5}$ Torr has been achieved. The films grown at $1 \times 10^{-2}$ Torr, however, showed a halo RHEED pattern, indicating the $\mathrm{LaAlO}_{3}$ was amorphous.

Figure 2 shows histograms of breakdown electric field of $\mathrm{LaAlO}_{3}$ epitaxial insulator grown at oxygen partial pressure of $1 \times 10^{-4}$ Torr evaluated by $\mathrm{Al} / \mathrm{LaAlO}_{3} / \mathrm{Nb}: \mathrm{SrTiO}_{3}$ capacitor structure before and after post annealing at $400^{\circ} \mathrm{C}$ for $2 \mathrm{~h}$ in atomospheric $\mathrm{O}_{2}$. The area of the $\mathrm{Al}$ electrode was $1.3 \times 10^{-3} \mathrm{~cm}^{2}$. For as-grown $\underline{\mathrm{LaAlO}}_{3}$, the values and the deviations of breakdown electric field were low and wide, $\underline{\text { respectively. For post annealed } \mathrm{LaAlO}_{3}, \text { in contrast, the values were improved to about } 2}$

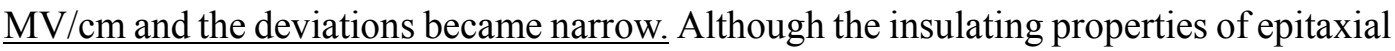
$\mathrm{LaAlO}_{3}$ even after post annealing were inferior to those of amorphous $\mathrm{LaAlO}_{3}[11]$, post annealing after $\mathrm{LaAlO}_{3}$ growth resulted in the improvement of the device yield.

We have also fabricated thin $\mathrm{LaAlO}_{3}$ films (50 nm thick). The surface morphology was step and terrace structure, indicating atomically flat surface, which was expected to result in fabricating well-defined insulator/semiconductor interface. Insulating properties, however, were not sufficient to operate FETs, i.e., very leaky, mainly due to the current through the grain boundaries in epitaxial $\mathrm{LaAlO}_{3}$. Thin $\mathrm{LaAlO}_{3}$ enhanced the quality of surface morphology, but did not meet the insulating properties required for a meaningful field effect device. 
Figure 3 shows FET characteristics of $\mathrm{TiO}_{2}$ FET with bottom-gate structure in air. $\mathrm{LaAlO}_{3}$ epitaxial insulator was grown at oxygen partial pressure of $1 \times 10^{-4}$ Torr and post annealed. Typical accumulation type FET actions were observed. Drain voltage $\left(V_{d}\right)$ - drain current $\left(I_{d}\right)$ curves clearly shows pinch-off points and current saturation. Gate voltage $\left(V_{g}\right)-I_{d}$ curves shows the on-to-off current ratio exceeding $10^{4}$. Leakage current ( $\left.I_{g}\right)$ was relatively high, but it was satisfactorily lower than the $I_{d}$. Field-effect mobility at saturated regime was $0.04 \mathrm{~cm}^{2} / \mathrm{Vs}$. The maximum carrier density deduced from the conductivity and the mobility was of the order of $10^{18} \mathrm{~cm}^{-3}$.

FET characteristics obtained in this study was inferior to that of top-gate device structure [12]. It seemed to be resulted from two factors; the quality of $\mathrm{TiO}_{2} / \mathrm{LaAlO}_{3}$ interface and the crystallinity of $\mathrm{TiO}_{2}$. Judging from surface morphology of epitaxial $\mathrm{LaAlO}_{3}$ insulator, $\mathrm{TiO}_{2} / \mathrm{LaAlO}_{3}$ interface was not atomically sharp. Thus, the interface was considered to contain many defects, which can act as trapping and scattering sites of carriers. Feild-effect carrier control is basically interface phenomena, thus the quality of the interface was critical. Moreover, the crystallinity of $\mathrm{TiO}_{2}$ active layers was not so high, because the crystallinity of $\mathrm{LaAlO}_{3}$ beneath the $\mathrm{TiO}_{2}$ was not so high, resulting in low carrier mobility. More improvement in quality of the epitaxial insulator, surface morphology and the crystallinity is, therefore, required, which further, leads to an improved responses to the ambient and light as mentioned below.

Figure 4 shows FET characteristics under light (Xe lamp, $150 \mathrm{~mW} / \mathrm{cm}^{2}$ ) and/or oxygen ambient. As shown in Fig. 4(a), under oxygen ambient, $I_{d}$ was slightly reduced 
compared with that under vacuum $\left(1 \times 10^{-6}\right.$ Torr $)$ irrespective of light illumination. This result was expected as the carriers may be trapped by adsorbed oxygen [13]. Under light illumination, $I_{d}$ was increased compared to that in dark, but still modulated by the gate voltage, indicating that field effect control was feasible under light illumination. These responses to ambient and light were reversible. The effects of light illumination almost diminished in a few tens of seconds after the turning off the light, while the effect of adsorbed oxygen remained for a few hours after evacuation. Figure 4 (b) shows $I_{d}$ response to alternating oxygen pressure under light illumination. Oxygen pressure was repeatedly changed from $1 \times 10^{-6}$ Torr to 760 Torr and vice versa. $I_{d}$ definitely responded to oxygen pressure. These results indicate the device potential for gas or light sensor application, where the dynamic range of the sensitivity can be controlled by gate voltage.

\section{Conclusion}

In summary, we have succeeded in fabricating the field-effect transistors based on $\mathrm{TiO}_{2}$ active channels with bottom-gate structure. One of the key points to achieve this is the growth of high-quality epitaxial insulator, which fulfills both insulating properties and high crystallinity, required for FET. Fairly good epitaxial $\mathrm{LaAlO}_{3}$ was obtained. Our bottom-gate devices showed typical transistor actions and clear responses to light and/or ambient, suggesting the sensor application with sensitivity tuned by the gate voltage.

\section{Acknowledgements}


This work was supported by CREST project from the Japan Science and Technology

Agency, and partially supported by Grant-in-Aid from the Ministry of Education, Culture,

Sports, Science and Technology of Japan (No. 17656115 and No.18750179). 


\section{References}

[1]. S. B. Ogale, Thin Films and Heterostructures for Oxide Electronics, Springer, New York, 2005.

[2]. C. H. Ann, S. Gariglio, P. Paruch, T. Tybell, L. Antognazza, J. -M. Triscone, Science 284 (1999) 1152-1155.

[3]. K. S. Takahashi, M. Gabay, D. Jaccard, K. Shibuya, T. Ohnishi, M. Lippmaa, J. -M. Triscone, Nature 441 (2006) 195-198.

[4]. T. Zhao, S. R. Shinde, S. B. Ogale, H. Zheng, T. Venkatesan, R. Ramesh, S. D. Sarma, Phys. Rev. Lett. 94 (2005) 126601.

[5]. T. I. Suzuki, A. Ohtomo, A. Tsukazaki, F. Sato, J. Nishii, H. Ohno, M. Kawasaki, Adv. Mater. 16 (2004) 1887-1890.

[6]. K. Nomura, H. Ohta, K. Ueda, T. Kamiya, M. Hirano, H. Hosono, Sciene 300 (2003) 1269-1272.

[7]. K. Ueno, I. H. Inoue, H. Akoh, M. Kawasaki, Y. Tokura, H. Takagi, Appl. Phys. Lett. 83 (2003) 1755-1757.

[8]. K. Shibuya, T. Ohnishi, T. Uozumi, T. Sato, M. Lippmaa, M. Kawasaki, K. Nakajima, T. Chikyow, H. Koinuma, Appl. Phys. Lett. 88 (2006) 212116.

[9]. M. Katayama, S. Ikesaka, J. Kuwano, Y. Yamamoto, H. Koinuma, Y. Matsumoto, Appl. Phys. Lett. 89 (2006) 242103.

[10].K Hashimoto, H. Irie, A. Fujishima, Jpn. J. Appl. Phys. 44 (2005) 8269-8285.

[11]. S. Yaginuma, J. Yamaguchi, K. Itaka, H. Koinuma, Thin Solid Films 486 (2005) 
218-221.

[12]. M Katayama, S. Ikesaka, J. Kuwano, H. Koinuma, Y. Matsumoto, Appl. Phys. Lett. submitted.

[13]. D. Kang, H. Lim, C. Kim, I. Song, J. Park, Y. Park, J. Chung, Appl. Phys. Lett. 90 (2007) 192101. 


\section{Figure captions}

Fig.1 (a) Schematic illustration of the device structure. (b) RHEED image, (c) AFM image $\left(1 \times 1 \mu \mathrm{m}^{2}\right)$, and (d) XRD patterns of epitaxial $\mathrm{LaAlO}_{3}$ fillm. The inset of (d) is a rocking curve of $\mathrm{LaAlO}_{3}(002)$ diffraction.

Fig. 2 Histograms of breakdown electric field of epitaxial $\mathrm{LaAlO}_{3}$ film (a) before and (b) after post annealing.

Fig. 3 Transistor characteristics of bottom-gate anatase FET.

Fig. 4 (a) $\mathrm{V}_{\mathrm{g}}-\mathrm{I}_{\mathrm{d}}$ curves of anatase $\mathrm{TiO}_{2}$ FET under various conditions. Open and filled markers denote under Xe light illumination and dark condition, respectively. Triangle and circle markers denote ambient condition; $1 \times 10^{-6}$ Torr and 760 Torr $\mathrm{O}_{2}$ ambient, respectively. (b) Drain current responses to oxygen pressure under Xe lamp illumination. 
(a)

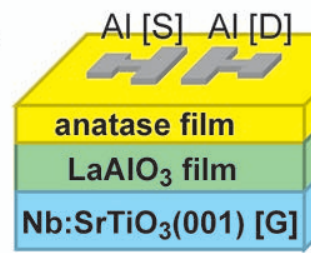

(c)

(b)

(d) $10^{7}$

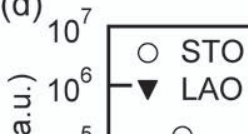

ذ FWHM $0.7^{\circ}$

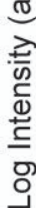

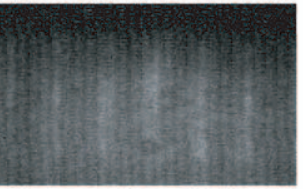

O

0

$\checkmark$

O

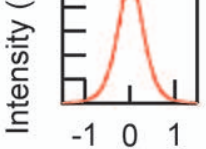

$\left.\right|^{\theta\left(^{\circ}\right)}$

$10^{1} \frac{10^{2}-}{20 \quad 40}$

60

80

100

$2 \theta\left({ }^{\circ}\right)$

Fig. 1 Katayama et al. 
(a) 20

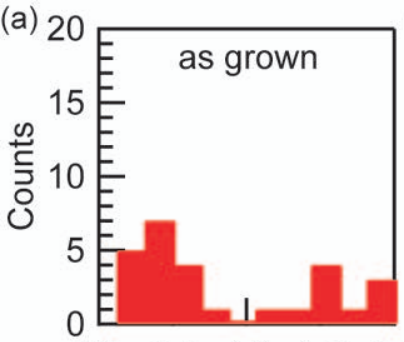

$\begin{array}{lllll}0.0 & 0.5 & 1.0 & 1.5 & 2.0\end{array}$

Electric field $(\mathrm{MV} / \mathrm{cm})$ (b)

\section{E post annealed}

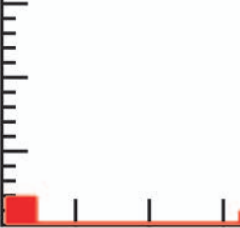

$\begin{array}{lllll}0.0 & 0.5 & 1.0 & 1.5 & 2.0\end{array}$ Electric field $(\mathrm{MV} / \mathrm{cm})$ 
(a)

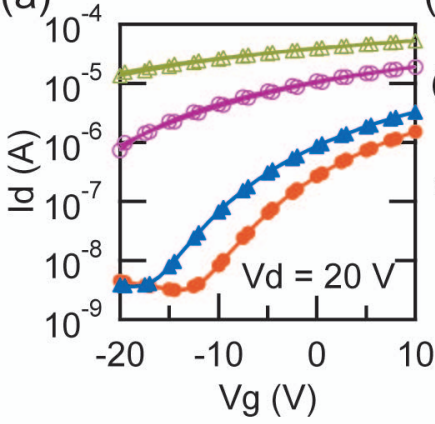

(b)

$\mathrm{PO}_{2}$ (Torr)

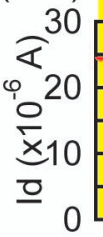

0
760760 $1 e-6 \quad 1 e-6 \quad 1 e-6$

760

$\mathrm{Vd}=20 \mathrm{~V}$

$\mathrm{Vg}=-25 \mathrm{~V}$

10

1000

Time (s)

Fig. 4 Katayama et al. 